\title{
Eventos científicos: da Pirâmide Reputacional aos círculos persuasivos
}

\author{
Maiko Rafael Spiess* \\ Marcos Antonio Mattedi**
}

Resumo: O artigo aborda o tema dos eventos científicos. Argumenta que os eventos científicos constituem uma estratégia de comunicação que afeta a comunidade científica de forma social, espacial e cognitiva. O texto operacionaliza conceitos e teorias sociológicas como forma de analisar os eventos científicos. Para isso, baseia-se em pesquisa bibliográfica e histórica. Está dividido em cinco partes principais: $i$. uma introdução com a problematização sociológica dos eventos científicos; ii. uma análise do processo de formação e desenvolvimento dos eventos científicos; iii. uma revisão crítica da abordagem sociológica do Programa Normativo de análise da atividade científica; iv. uma revisão do Programa Discursivo dos eventos científicos; v. uma conclusão, que oferece subsídios para desenvolvimento da abordagem sociológica dos eventos científicos. 0 artigo conclui que os eventos científicos podem ser vistos como espaços que refletem a estratificação da comunidade científica, mas também como arenas de persuasão que são mobilizadas no processo de construção do conhecimento.

Palavras-chave: Eventos científicos. Comunicação científica. Comunidade científica. Hierarquia. Sociologia da ciência.

\section{Scientific meetings: from the reputational pyramid to the persuasion cycles}

Abstract: The paper addresses the topic of scientific meetings. It argues that scientific meetings are a communication strategy that affects the scientific community socially, spatially, and cognitively. The text employs sociological theories as a way of analyzing scientific events. For this, is based on bibliographic and historical research. It is divided in five sections: $i$. an introduction on the sociological approach on scientific meetings; ii. an account on the rise and development of scientific meetings; iii. a critical review on the Normative Program sociological approach of scientific activities; iv. a critical review on the Discursive Program of scientific meetings; $v$. a conclusion section, with elements for a sociological approach of scientific meetings. The paper concludes that scientific meetings are spaces that reflect the stratification within the scientific community, but also as persuasion arenas mobilized for knowledge construction.

Keywords: Scientific meetings. Scientific communication. Scientific community. Hierarchy. Sociology of science.

\author{
* Maiko Rafael \\ Spiess é formado \\ em ciências sociais \\ pela Universidade \\ Regional de \\ Blumenau (2007), \\ mestre (2010) e \\ doutor (2014) em \\ política científica \\ e tecnológica \\ pela Universidade \\ Estadual de \\ Campinas (Unicamp); \\ é professor efetivo \\ no Departamento \\ de Ciências Sociais \\ e Filosofia, na \\ Universidade \\ Regional de \\ Blumenau, membro \\ coordenador do \\ Núcleo de Estudos da \\ Tecnociência (Furb), \\ Blumenau, Santa \\ Catarina, Brasil. \\ Orcid: 0000-0003- \\ 1008-7739. \\ <mspiess@furb.br>. \\ ** Marcos Antonio \\ Mattedi é bacharel \\ em ciências sociais \\ pela Universidade \\ Regional de \\ Blumenau (1991), \\ mestre em sociologia \\ política pela \\ Universidade Federal \\ de Santa Catarina \\ (1994) e doutor \\ em ciências sociais \\ pela Universidade \\ Estadual de \\ Campinas (1999). \\ É professor titular \\ do Programa de \\ Pós-Graduação em \\ Desenvolvimento \\ Regional da
}


Universidade

Regional de

Blumenau, Santa

Catarina, Brasil;

coordenador do

Núcleo de Estudos da

Tecnociência (NET).

Orcid: 0000-0002-

0046-7853.

<mattediblu@gmail.

com>.

\section{Introdução}

$\Lambda$ atividade científica não é apenas uma relação do cientista com o mundo, mas também uma relação do cientista com outros cientistas. Isso significa que produzir, transmitir ou aplicar o conhecimento científico constitui uma atividade social. Portanto, para comunicar suas descobertas científicas, o cientista precisa entrar em contato com outros cientistas. Afinal, a relação entre emissores e receptores de informações é sempre socialmente contextualizada. Tanto o conhecimento codificado como a expertise tácita entre cientistas dependem, fundamentalmente, da mediação estabelecida entre o veículo empregado e o público-alvo (Meadows, 1999). Essa mediação não afeta apenas a forma, mas ainda a quantidade de informação em circulação na comunidade científica, indica que a capacidade de comunicação caracteriza a ciência como atividade marcadamente social. Neste sentido, um dos aspectos menos conhecidos da dimensão coletiva da ciência, em geral, e da comunicação científica, em particular, são os eventos científicos.

Os eventos científicos são uma das formas mais utilizadas de comunicação científica. Tem a função de facilitar a difusão de informações na comunidade científica e, consequentemente, o processo de atualização dos cientistas. No que se refere ao fluxo de informações, apresentam duas características principais:

i. oralidade da troca de informações por meio de apresentações e discussões; e

ii. fluidez e flexibilidade dos contatos diretos e interpessoais.

No que diz respeito à organização, concentram-se num curto espaço de tempo e abrangem duas principais formas de diferenciação, externa e interna:

i. congressos, conferências, colóquios, seminários e outros eventos congêneres;

ii. apresentações e sessões plenárias, participação em workshops e mesas redondas, leitura e avaliação de pôsteres.

Do ponto de vista relacional, podem ser vistos como uma espécie de "feira cognitiva": um lugar onde são apresentados, oferecidos e construídos produtos cognitivos. Pode-se se dizer, portanto, que os eventos científicos se caracterizam pela transitividade.

Os eventos científicos têm uma longa história. Constituem a forma mais tradicional de comunicação científica. Os primeiros registros remontam ao século XVII, como 
ilustram as reuniões realizadas pela Accademia Nazionale dei Lincei, em 1603; pela Accademia degli Investiganti, em 1650; e pela Royal Society, em 1660. Com o tempo, esses encontros ocasionais foram se tornando regulares por causa das necessidades surgidas pelo estabelecimento de procedimentos institucionais da pesquisa científica. Consequentemente, com o aumento do número de cientistas, o volume e a variedade de informações transmitidas por meio de eventos científicos se amplia exponencialmente. À medida que a atividade científica se massifica verifica-se que os eventos científicos foram assumindo novas funções. Hoje as comunicações orais dispõem de formas diferentes de realização, tanto no que se refere ao padrão coletivo de organização, quanto às características técnicas da exposição. A consequência mais evidente deste processo é que eventos científicos parecem acompanhar a tendência de especialização científica.

Ocorre que os eventos não são apenas uma assembleia de pessoas reunidas para a transmissão de informações. Por um lado, a participação em eventos científicos constitui uma ruptura com a rotina cotidiana das atividades de laboratório, da sala de aula e do escritório; por outro, representa uma continuidade, na medida em que as conferências são uma espécie de extensão do local de trabalho. Costumam ser considerados como uma oportunidade para que os membros de um coletivo forjem relacionamentos, estabeleçam estatutos e definam papéis dentro da comunidade científica. Mais precisamente, são espaços nos quais o reconhecimento é distribuído, a reputação é combatida e onde a dinâmica cognitiva é promulgada e projetada. Embora não haja um critério geral para classificar os eventos científicos, é possível identificar uma série de características comuns, tais como:

i. registro burocrático dos participantes;

ii. realização em ambiente fisicamente isolado;

iii. regras particulares de conduta;

iv. cronograma com programa definido de atividades; e

v. interação intensa entre os participantes.

Além disso, os eventos científicos possuem uma grande variação segundo sua área de pesquisa. Em algumas disciplinas, os eventos científicos são considerados como ponto de chegada da apresentação da produção do conhecimento científico; em outras, os eventos científicos são concebidos como ponto de partida para o desenvolvimento da pesquisa científica. Assim, enquanto em alguns eventos científicos são apresentadas descobertas e inovações, em outros constituem espaço de iniciação e experimentação. Por exemplo, na área de informática, os eventos cientí- 
ficos são altamente valorizados em termos cognitivos (Vardi, 2009; Hermenegildo, 2012), já na área de sociologia, são menos importantes (Powell, 1985). Verifica-se uma variação não somente disciplinar, mas também paradigmática entre os eventos científicos. Por isso, as fronteiras entre o público e o privado, portanto, a autonomia da comunidade científica varia de evento para evento. Neste contexto, emerge a questão de como os eventos são citados e, consequentemente, como são avaliados.

Apesar da relevância teórica e prática, a abordagem dos eventos científicos permanece, curiosamente, um objeto de estudo pouco explorado na sociologia da ciência. Por isso, um conjunto de questões centrais - tanto para o desenvolvimento da atividade científica quanto para avaliação da produtividade como, por exemplo, a quantidade de eventos que são realizados anualmente, a justificação, como são financiados e quantos recursos movimentam, que tipo de conhecimento mediam e como são avaliados, onde são realizados e quantos pesquisadores participam permanecem abertas. Nosso argumento é que os eventos constituem, simultaneamente, marcações cognitivas, sociais e espaciais da comunidade científica. Por isso, para tratar essa problemática é preciso considerar três questões principais:

i. Dimensão cognitiva - quais são os tipos de conhecimento produzidos nos eventos científicos?

ii. Dimensão social - quais são os padrões de interação entre os participantes?

iii. Dimensão espacial - quais são os efeitos da distribuição espacial dos eventos científicos?

FIGURA 1

AS TRÊS DIMENSÕES DOS EVENTOS CIENTíFICOS

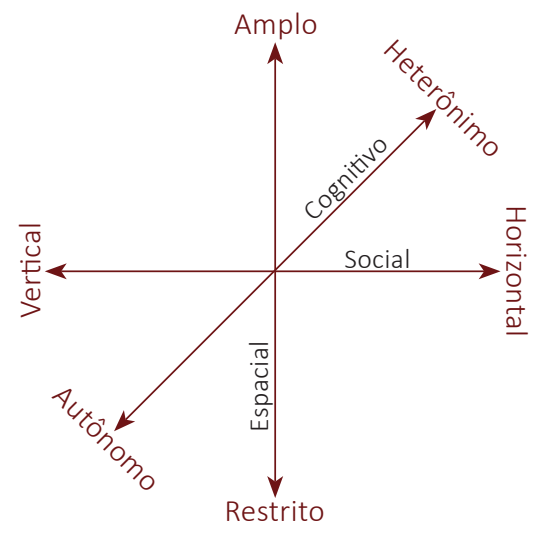

Fonte: elaboração própria. 
A relação entre as dimensões social, espacial e cognitiva pode ser analisada por meio de várias abordagens sociológicas da ciência. Por exemplo, pode ser interpretada considerando os eventos científicos como espaço de disputas por meio do conceito de "campo científico" (Bourdieu, 1976; 2001; 2009; 2011); pode ser interpretada também com base na consideração dos interesses (Bloor, 1976; Shapin, 1979; Collins \& Pinch, 2003). Os eventos científicos podem ser caracterizados recorrendo aos subsídios extraídos dos estudos de laboratório (Latour \& Woolgar, 1997; Knorr-Cetina, 1981; Lynch, 1985). Como o conceito de campo científico já foi aplicado anteriormente para o caso dos eventos (Silveira, Bufrém \& Caregnato, 2015), a abordagem apresentada aqui se concentra analiticamente em duas estratégias principais:

i. Programa normativo: considera as atividades científicas por meio das disposições interativas que se estabelecem entre os cientistas; e

ii. Programa discursivo: considera as atividades científicas por meio propensões retóricas empregadas pelos cientistas.

Ao considerar essas duas estratégias, o artigo apresenta uma aproximação inicial, de caráter teórico-conceitual, ao tema. Por um lado, mobiliza abordagens previamente estabelecidas como forma para apresentar questionamentos e hipóteses relacionados aos eventos científicos. Nesse sentido, não se trata de uma adesão estrita, dogmática, mas um recurso analítico. Por outro lado, o artigo busca identificar possíveis fenômenos recorrentes a respeito dos eventos científicos que possam se converter em agendas de pesquisa, visando aprofundar o trabalho empírico sobre o tema. Dessa forma, procura contribuir para aprofundar discussões a respeito dos eventos científicos como forma de comunicação e construção de conhecimento, que são ainda preliminares na sociologia da ciência e estudos sociais da ciência e da tecnologia no Brasil.

Portanto, o objetivo deste texto é formular uma abordagem tridimensional dos eventos científicos. Esta abordagem baseia-se em três procedimentos:

i. identificação dos atributos recorrentes;

ii. aplicação dos programas normativo e discursivo; e

iii. equivalência de semelhanças e diferenças.

Para apresentar esta abordagem o texto foi dividido em cinco partes: na primeira, discute-se a problematização inicial dos desafios da sociologia dos eventos científicos; na segunda parte, é apresentado o processo de formação e desenvolvimento 
histórico dos eventos; na terceira parte, são identificadas as implicações da realização do programa normativo, destacando o duplo efeito Mateus que os eventos científicos desencadeiam na comunidade científica; na quarta parte, apresentam-se os resultados da interpretação do programa discursivo, enfatizando os círculos persuasivos; para finalizar, registra-se um conjunto de subsídios teóricos baseados numa crítica da "eventolarquia", por meio da proposta de uma reflexividade experimental.

\section{O processo de formação e institucionalização dos eventos científicos}

Os eventos científicos desempenham um papel central na atividade científica. A importância dos eventos está relacionada não apenas à comunicação de descobertas, mas também ao processo de socialização. A motivação para a organização de eventos científicos pode ser muito variada, abrangendo, por exemplo, o compartilhamento de ideias, o estabelecimento de parcerias, a formação de uma especialidade de pesquisa etc. Por isso, podem ser formados por especialistas e não especialistas, isso em razão de os eventos científicos se modificarem ao longo do tempo. Este contínuo processo de transformação reflete as próprias transformações da comunidade científica. À medida que a comunidade científica se institucionaliza, o processo de comunicação se massifica e, consequentemente, a função e o padrão de organização dos eventos científicos. Neste sentido, o processo de institucionalização dos eventos científicos pode ser dividido em três fases principais:
i. Período de formação;
ii. Período de consolidação; e
iii. Período de massificação.

Período de formação

Nos dias de hoje, os eventos científicos são indissociáveis das associações ou organizações de pesquisadores profissionais que os organizam e patrocinam. Todavia, é possível supor que os encontros de cientistas antecedem o surgimento das primeiras sociedades científicas. Por exemplo, alguns historiadores da ciência apontam que o surgimento da Royal Society inglesa foi antecipado por um "colégio invisível" - possivelmente composto de notáveis como Robert Boyle, John Wallis e John Wilkins (Webster, 1974) -, envolvidos tanto com os aspectos experimentais quanto com os interesses políticos dos "filósofos naturais" - os cientistas da época. Nesse sentido, os encontros organizados para a replicação de experimentos, como a bomba de ar de Boyle, não representavam apenas a divulgação de avanços técnicos, 
mas também a criação e o estabelecimento de um "espaço social" de debate aberto para a burguesia diletante no contexto da Inglaterra pós-restauração (Shapin \& Schaffer, 1985).

Com a efetiva criação das primeiras sociedades científicas, os eventos da comunidade científica encontraram uma base institucional mais estável para o seu desenvolvimento e a sua recorrência. Tanto a Royal Society inglesa (criada em 1663) como a Académie des Sciences (criada em Paris, em 1666) representavam assim uma mudança significativa em relação à organização dos cientistas, introduzindo gradativamente normas sociais relacionadas à publicação de resultados experimentais, ao reconhecimento de contribuições individuais e à abertura ao escrutínio e crítica dos pares (Ben-David, 1974). No caso particular da Académie des Sciences, a instituição era parcialmente patrocinada e legitimada pelo regime de Luís XIV, significando simultaneamente a legitimação institucional e o controle estatal da nascente comunidade científica. Assim, os encontros que anteriormente eram pouco estruturados passam a ter a regularidade e o suporte institucional garantidos pelas sociedades científicas.

Mais precisamente, os eventos científicos constituem a primeira forma de comunicação científica oficial. Os primeiros registros dizem respeito ao Summer Science Exhibition, organizado pela Royal Society of London, em 1778, que teve lugar na residência de seu presidente, Joseph Banks. Sua realização visava fortalecer os vínculos de comunicação mediante a troca direta de informações e experiências entre cientistas. Apesar de seu caráter informal, com o tempo acabou se tornando regular, mantendo a mesma denominação até o presente. Concomitantemente, devido às limitações de locomoção e circulação, por muito tempo estes encontros permaneceram espacialmente localizados, reunindo um pequeno número de pesquisadores. Em função das dificuldades de circulação das informações escritas, os eventos científicos se converteram nas formas mais importantes de disseminação das descobertas científicas.

Portanto, as reuniões científicas representam a gradual organização cognitiva e política da comunidade científica que aparece ao lado da formação das sociedades científicas pioneiras surgidas na França, Inglaterra e Alemanha. Por um lado, as reuniões científicas materializam a institucionalização da comunidade científica em termos de vínculos e compromissos (Gribbin, 2007); por outro, constituíam uma oportunidade para a apresentação de novas áreas de conhecimento e identificação de novas linhas de desenvolvimento. Pode-se dizer que se caracterizavam por relações fortemente pessoais, onde o "pré-conhecimento" é decisivo no processo de produção do conhecimento. Isso significa que, em seus primórdios, os eventos 
científicos podem ser vistos como uma espécie de peer review informal (Carpi \& Egger, 2009). Neste sentido, os eventos científicos não são apenas assembleias de informação, mas espaços coletivos de mediação da autoridade.

\section{Período de consolidação}

Com a criação da universidade moderna e a profissionalização das atividades científicas, verifica-se uma transformação do padrão de organização da comunidade científica. A implantação da Humboldt-Universität zu Berlin (HU-Berlin), em 1809, inicia um novo patamar na produção do conhecimento científico. A pesquisa científica se concentrava nos institutos vinculados à universidade. Os institutos consistiam em organizações burocráticas permanentes, que possuíam seus próprios edifícios, suas equipes docentes e de apoio. Por um lado, a pesquisa científica, que era desenvolvida nas academias de ciência, passa ser realizada nos laboratórios universitários; por outro, os pesquisadores passam a ter uma formação regular e os professores uma carreira estável (Ben-David, 1974). Isso significa que a instrução sistemática e a divisão do trabalho se tornaram fatores importantes do desenvolvimento científico. Consequentemente, a organização universitária transformou a comunidade científica devido à profissionalização e à especialização disciplinar.

A transformação nos padrões de organização da comunidade científica se evidencia também nas formas de comunicação científica. Estas mudanças provocaram um aumento da produção científica em termos de publicações e, consequentemente, nos canais de comunicação (Hagstrom, 1965). Isso significa que, com a profissionalização das carreiras de pesquisa, com o surgimento do laboratório universitário e a instituição de processos padronizados ampliam-se também as escalas formal e informal de circulação de informações. Mais precisamente, com a profissionalização das atividades científicas, se profissionalizam também os processos de comunicação científica. Por um lado, cresce o número e a regularidade dos periódicos científicos; por outro, intensifica-se a troca de informações entre subcomunidades disciplinares. Essa troca de informações entre especialidades estabelece os padrões de organização dos eventos científicos modernos. Neste sentido, os eventos científicos aumentam de tamanho e apresentam uma maior variação espacial.

Em outras palavras, a crescente profissionalização da comunidade científica representou um aumento da importância e centralidade dos eventos. Um exemplo pioneiro foi o Congresso de Karlsruhe, realizado na cidade alemã de mesmo nome, em setembro de 1860. Sua relevância é dupla: por um lado, o encontro de químicos é considerado o primeiro evento científico verdadeiramente internacional, reunindo cerca de 140 profissionais de 12 países europeus (Ihde, 1961); por outro, represen- 
tou um marco importante no campo da química, sobretudo do ponto de vista das tentativas iniciais de estabelecimento de padrões conceituais e de notação entre os praticantes. O congresso encerrou-se sem consenso, em parte pelo conflito entre veteranos e pesquisadores mais jovens (Everts, 2010), no entanto, as discussões iniciadas realizadas em Karlsruhe a respeito do peso atômico dos elementos químicos abriram caminho para diferentes desenvolvimentos posteriores no campo da química e da física atômica (Nye, 1984).

O papel fundacional e normativo dos eventos científicos se repetiu também em outros campos do conhecimento. Assim, o Congresso Internacional de Botânica (realizado pela primeira vez em Bruxelas, em 1864) além de representar a crescente especialização da comunidade das ciências naturais, também é o espaço onde a nomenclatura internacional de botânica é definida e revisada desde 1867 (Stafleu, 1970). Em muitos casos, eventos científicos foram organizados para discutir temas emergentes dentro de determinadas áreas do conhecimento, evidenciando tanto as respostas cognitivas como os posicionamentos institucionais dos cientistas a novos problemas. Por exemplo, a sessão de encerramento do I Congresso Internacional sobre a Malária (Roma, 1925) foi concluída com uma moção para a criação de um Instituto Internacional de Malariologia (British Medical Journal, 1925). Assim, é possível notar que os eventos são marcadores da gradual profissionalização da ciência, mas também do processo de internacionalização da comunidade científica.

Período de massificação

Após a Segunda Guerra Mundial, verifica-se uma transformação profunda no padrão de organização da comunidade científica. Esse processo se caracteriza, por um lado, pela criação de políticas governamentais e pelo aumento progressivo de recursos investidos; por outro, pelo redimensionamento das fronteiras institucionais da comunidade científica e, consequentemente, da pesquisa científica (Price, 1963). Essas inovações tiveram como principais consequências o aumento do volume e da variedade de informações produzidas, bem como, a maior dinamização e especialização da atividade científica em termos disciplinares. Mais precisamente, a modificação dos parâmetros de organização teve como consequência a transformação da dinâmica de integração da comunidade científica e da estrutura de reputação, sintetizada na famosa expressão "publish or perish" (Garfield, 1996). Consequentemente, os parâmetros de controle e avaliação da comunidade científica também se modificam (Mattedi \& Spiess, 2017).

No contexto cognitivo da big science opera-se uma mudança de escala na produção científica. Como consequência dessa mudança, também ocorre um processo 
de massificação da comunicação científica. O conhecimento científico cresce em escala e converte-se numa comodity do processo de inovação tecnológica. Neste sentido, verifica-se não somente o aumento de publicações, mas também uma diversificação dos meios de divulgação. Com o avanço da eletrônica e da ciência da computação, o mercado editorial científico dá origem às grandes corporações como, por exemplo, Elsevier, Wiley-Blackwell, Springer, Sage etc. Essas transformações no padrão de organização da comunidade redimensionam o propósito, a abrangência e o significado dos eventos científicos. Mais precisamente, os eventos científicos se tornam mais regulares, maiores e especializados. Neste sentido, de forma muito mais explícita que os periódicos, os eventos científicos convertem-se em "sismógrafos" da dinâmica de desenvolvimento científico.

Paradoxalmente, o impacto da massificação dos eventos científicos não é amplamente discutido pela comunidade científica. Normalmente, do ponto de vista qualitativo, a importância dos eventos é avaliada apenas em debates realizados por comunidades específicas, por ocasião de datas comemorativas, como, por exemplo, o colóquio especial comemorativo "A Associação Nacional de Pós-Graduação e Pesquisa em Ciências Sociais (Anpocs) e as ciências sociais nos últimos 40 anos", realizado no quadragésimo aniversário da associação. Do ponto de vista quantitativo, é possível observar que o impacto dos eventos científicos já foi analisado, por exemplo, a partir das ferramentas da bibliometria e cientometria (Drott, 1995; Glänzel, Debackere, Thijs \& Schubert, 2006; Lisée, Larivière \& Archambault, 2008). Existem, assim, estudos que buscam avaliar a qualidade das conferências a partir de indicadores de citações bibliográficas (Martins et alii, 2010), da utilização dos trabalhos apresentados como referências bibliográficas (Michels \& Fu, 2014), da publicação em revistas de artigos produzidos a partir de comunicações em eventos (Aleixandre-Benavent et alii, 2009), dentre outros.

No entanto, em linhas gerais, esse tipo de discussão costuma estar concentrado em periódicos especializados como Scientometrics, Journal of the American Society for Information Science and Technology (Jasist) ou similares. Também não são muito numerosas as pesquisas destinadas a compreender as motivações e experiências dos frequentadores e organizadores de eventos científicos. Nesse aspecto em particular, seriam necessários estudos empíricos, como o de Hayashi e Guimarães (2016), para mensurar e avaliar as disposições individuais e as tendências coletivas em relação aos eventos científicos. Além disso, seria possível aprofundar a investigação sobre o funcionamento e a eficácia dos eventos científicos como espaços de colaboração, especialmente se considerados os diferentes modos de produção científica (Hessels \& van Lente, 2008) e as condições da ciência pós e extra-acadêmica (Ziman, 1996; 2000). Assim, é possível afirmar que o conhecimento siste- 
matizado sobre a fase atual dos eventos e o seu impacto científico ainda está em construção, podendo avançar teórica e empiricamente.

Todavia, as práticas, a abrangência e o foco dos eventos variam enormemente e estima-se que sua realização, organização e documentação converteu-se em uma atividade econômica significativa. Neste sentido, é possível destacar:

i. a consolidação da prática de publicação de anais (ou "proceedings") dos eventos, contendo resumos ou artigos completos, organizados pelas entidades realizadoras ou grupos editoriais comerciais (Thomson Reuters, Springer etc.);

ii. a profissionalização da organização, por meio da crescente atuação de entidades privadas ou sem fins lucrativos (Gordon Research Conferences, Keystone Symposia) dedicadas à organização prática e logística dos eventos, ou surgimento de diretórios de eventos na Internet (Nature Events Directory);

iii. o aumento da variedade dos eventos, que, além de integrarem mais membros da comunidade científica por meio de sessões paralelas de pôsteres, painéis, oficinas e transmissão via streaming, também passaram a incluir leigos em conferências mistas ou "de consenso" (Fixdal, 1997).

QUADRO 1

AS FASES DOS EVENTOS CIENTÍFICOS

\begin{tabular}{|l|c|c|c|}
\hline \multicolumn{1}{|c|}{ Fases } & Formação & Consolidação & Massificação \\
\hline Características & Amador & Profissional & Fragmentado \\
\hline Tipo de evento & Restrito & Ampliado & Especializado \\
\hline Tipo de interação & Exclusivo & Hierárquico & Conflitivo \\
\hline Tipo de comunicação & Oral & Oral/impresso & Oral/impresso/eletrônico \\
\hline Fonte: elaboração própria.
\end{tabular}

Os diferentes períodos históricos apresentados descrevem as mudanças ocorridas nos eventos científicos. Em linhas gerais, refletem os processos de expansão, especialização e profissionalização da ciência. Mais precisamente, os eventos científicos são parte fundamental dos processos de demarcação da comunidade científica em relação ao contexto social, de constituição de divisões disciplinares internas, de formas de organização, de padrões de comunicação e de consolidação de valores compartilhados. Além disso, expressam o impacto de mudanças sociais e tecnológicas mais amplas na prática científica, como o aumento de recursos para 
pesquisa, diminuição dos custos e de tempo para o deslocamento de pesquisadores, bem como a introdução da comunicação eletrônica. Neste sentido, os eventos apresentam três atributos analíticos:

$$
\begin{aligned}
& \text { i. realização sazonal; } \\
& \text { ii. participação hierarquizada; e } \\
& \text { iii. organização disciplinar. }
\end{aligned}
$$

Portanto, é possível afirmar que quanto mais institucionalizados e recorrentes os eventos científicos, mais especializada e complexa é a organização da atividade científica.

\section{O programa normativo: o duplo efeito Mateus}

Como forma de comunicação, os eventos científicos exprimem a organização da comunidade científica. Isso significa que, para entender os eventos científicos, é preciso compreender a organização e o funcionamento da comunidade científica. Contextualmente, os eventos científicos não são apenas uma troca de informação entre os cientistas: os cientistas, ao se comunicarem, produzem e reproduzem os valores predominantes na comunidade científica, uma vez que, por um lado, os cientistas sempre se comunicam dentro de uma estrutura social e, por outro, ao se comunicarem, reforçam essa estrutura. Ou seja, a comunicação é realizada segundo regras institucionais que reforçam a própria instituição. Existem muitas formas de examinar a relação entre os eventos científicos e a comunidade científica, na medida em que a comunidade científica é integrada de forma normativa, cognitiva e transacional (Mattedi \& Spiess, 2010). Dentre as principais estratégias, destacam-se os subsídios extraídos do Programa Mertoniano.

O Programa Mertoniano ocupa-se dos cientistas enquanto profissionais, destacando seus valores e suas normas. Além de ser reconhecido como um dos sociólogos mais influentes do século XX, o trabalho específico de Robert K. Merton sobre a sociologia da ciência é considerado como o ponto de partida para a reflexão sobre a organização da comunidade científica (Cole, 2004). Seu interesse pelo tema remonta à sua tese de doutorado, Science, society, and technology in Seventeenth Century England (1938), e se estende para uma série de artigos influentes tais como: "The normative structure of science" (publicado em 1942 e reeditado em 1973), "Priorities in scientific discovery: a chapter in the sociology of science" (1957) ou "The Matthew effect in science" (1968). Progressivamente, Merton e seus seguidores 
consolidaram uma abordagem sociológica internalista, focada nos cientistas, seus valores, suas normas e práticas institucionalizadas.

Na perspectiva mertoniana, a autonomia da comunidade de cientistas em relação ao contexto social mais amplo é garantida por sua adesão a um ethos específico, que estrutura e orienta a prática científica. Ou seja, imperativos morais compartiIhados condicionam as práticas coletivas dos cientistas (Merton, 1970), ao mesmo tempo em que demarcam a distinção entre a comunidade científica e o restante da sociedade. Os valores compartilhados seriam centrais para a resolução de conflitos por prioridades em descobertas científicas (Merton, 1957), ou para a atribuição de reconhecimento e recursos. Nesta direção, Merton (1968) descreveu o efeito Mateus: a comunidade científica é, notadamente, um sistema estratificado, onde as recompensas são desproporcionalmente distribuídas de acordo com a reputação individual. A organização da ciência faz com que as contribuições de cientistas notórios e premiados tenham uma repercussão ampliada, enquanto os cientistas menos estabelecidos sejam frequentemente ignorados e desconsiderados.

Porém, essa diferenciação alicerça uma propriedade coletiva da comunidade científica e não um atributo individual dos cientistas. Mais precisamente, quanto mais institucionalizada a comunidade científica, maior o processo de estratificação das atividades científicas. No caso dos eventos científicos, essa característica da comunidade científica se manifesta de forma dupla. Por um lado, os eventos científicos hierarquizam os cientistas segundo a posição que ocupam na estrutura de apresentação; por outro, com relação a reputação dos lugares onde os eventos são realizados (Momm, 2017). Isso significa que os eventos científicos fixam, ao mesmo tempo, uma diferenciação entre cientistas, mas também entre lugares. Neste sentido, os eventos científicos compreendem um processo relacional que estrutura a comunidade científica tanto do ponto de vista social - superiores e inferiores -, quanto do ponto de vista espacial - centro e periferia. Portanto, essa relação se expressa em duas formas principais de estratificação:

i. hierarquia interna; e

ii. hierarquia externa.

A hierarquia interna diz respeito a posição que os cientistas ocupam na estrutura de comunicação e organização dos eventos científicos. Todos os eventos científicos se estruturam internamente segundo a Pirâmide Reputacional. A Pirâmide Reputacional superpõe papéis diferenciados dentro do evento científico, segundo critérios de relevância política e reconhecimento acadêmico. Neste sentido, os participantes se diferenciam em três grupos principais: 
FIGURA 2

A PIRÂMIDE REPUTACIONAL DOS EVENTOS CIENTÍFICOS

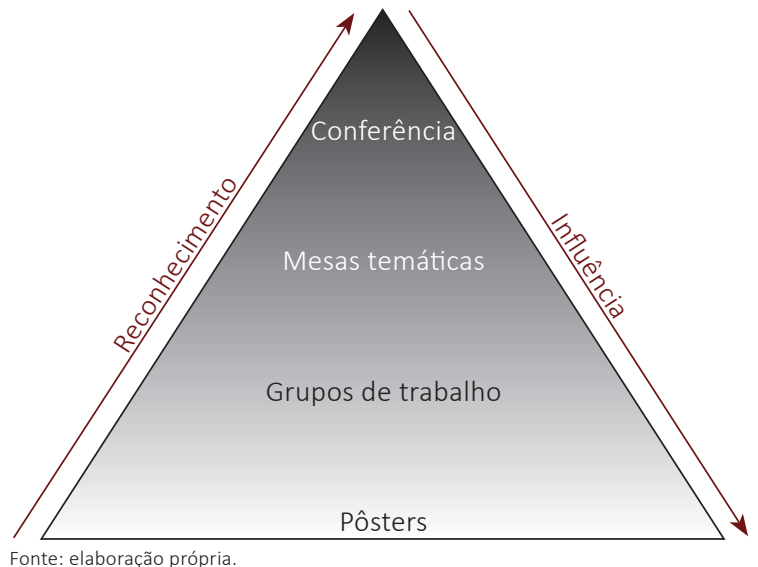

i. Prestígio amplo: cientistas de reconhecimento internacional que, frequentemente, são convidados para as conferências de abertura;

ii. Prestígio intermediário: cientistas convidados/selecionados para a participação das mesas temáticas e coordenação das atividades do evento; e

iii. Prestígio restrito: cientistas que concentram suas comunicações e participações nos grupos de trabalhos.

Essa hierarquia atua de modo que cada participante desempenhe seu papel correspondente à autoridade conferida à sua posição: quanto maior o prestígio, mais ampla a comunicação (Söderqvist \& Silverstein, 1994).

A Pirâmide Reputacional manifesta-se também na organização do evento, através de uma profunda divisão do trabalho. De um lado, situa-se uma "oligarquia acadêmica" que coordena, escolhe e formata política e cognitivamente o evento; de outro, um amplo conjunto de suporte, muitas vezes designado de "equipe de apoio", que executa as tarefas operacionais. Em primeiro lugar, essa diferenciação tende a variar em função da institucionalização e prestígio do próprio evento na comunidade científica; em segundo, em termos de gratificações e recompensas relativas ao reconhecimento. Neste sentido, quanto mais consolidado o evento, mais profissionalizada a sua organização. Isso significa que mais burocrática e impessoal serão as relações entre os organizadores e os operadores do evento. A reputação se converte em influência cognitiva e política. Ou seja, organizar o evento é determinar os 
papéis que os cientistas ocuparam na estrutura de comunicação. Neste sentido, os eventos possibilitam dois padrões de interação:

$$
\begin{aligned}
& \text { i. interação estruturada; e } \\
& \text { ii. interação não estruturada. }
\end{aligned}
$$

A interação estruturada diz respeito às formas de comunicação fechadas. Mais precisamente, compreende as mediações que se efetuam segundo os padrões estabelecidos pelas conferências, mesas temáticas, pelos grupos de trabalho, pôsteres etc. Esse tipo de comunicação é altamente padronizado, tanto em termos de tempo de exposição como em relação à forma de participação. Envolve, fundamentalmente, canais de comunicação oral e visual. Sua função interacional constitui a criação de um vínculo entre a visibilidade da pesquisa e a carreira de cientistas individuais. Baseia-se numa distribuição desigual do tempo de comunicação e, consequentemente, da atenção entre o comunicador e o espectador em termos do tempo de fala e dimensão da audiência. Apresentam uma relação inversamente proporcional entre tempo de comunicação e comentários: quanto maior a reputação do cientista, menores os comentários críticos. Consequentemente, os detentores de maior reputação geralmente obtêm mais espaço nos eventos, independentemente da originalidade de sua comunicação.

A interação não estruturada nos eventos científicos se caracteriza pelas oportunidades de contato e diálogo que ocorrem paralelamente à programação oficial. São exemplos dessa forma de interação as conversas nos corredores, confraternizações e contatos profissionais extraoficiais. Trata-se de uma forma de comunicação mais difusa e menos estruturada pelas dimensões formais dos eventos. Por um lado, podem ser oportunidades para um pesquisador de prestígio restrito interagir com profissionais localizados no topo da Pirâmide Reputacional, contornando os obstáculos da estrutura dos eventos; por outro, a suspensão das normas formais pode representar expectativas conflitantes em relação aos papéis sociais, como nos casos de favorecimento baseado em critérios ou indicações pessoais. Neste sentido, as interações não estruturadas representam a ambivalência sociológica (Merton, 1979) dos eventos, pois sua estratificação interna cria as próprias condições para os desvios normativos e as assimetrias políticas.

A hierarquia externa dos eventos científicos se evidencia na expressão territorial do efeito Mateus. Em outras palavras, é possível afirmar que a localização dos eventos científicos mais prestigiados e formalizados costuma coincidir com a localização dos principais locais produtores de conhecimento dentro de um campo ou especialidade científica. Este fenômeno estabelece uma relação hierárquica desigual entre o 
centro, a semiperiferia e a periferia da atividade científica. Os centros da produção científica são dotados de maiores recursos, maior produção científica e, portanto, maior reputação, credibilidade e anterioridade. Assim, os profissionais localizados em posições periféricas costumam valorizar os eventos centrais, convergindo para as regiões de maior importância em busca de legitimidade e oportunidades. Os pesquisadores estabelecidos localizados nas regiões centrais, no entanto, dificilmente costumam comparecer a eventos periféricos, a não ser para assumir uma posição privilegiada na Pirâmide Reputacional (por exemplo, como conferencista de abertura).

Portanto, é possível supor que existem diferenças importantes entre eventos centrais e periféricos. Em particular, os eventos podem ser diferenciados por meio de sua importância como espaço de produção e circulação do conhecimento, e da organização política da comunidade científica. Dessa forma, os eventos centrais são o espaço para a divulgação de teorias, descobertas e resultados pioneiros, isto é, uma arena para a discussão da "ciência de ponta". Representam um espaço de direcionamento da comunidade científica dos pontos de vista interno (coesão e direcionamento disciplinar e cognitivo) e externo (posicionamento político coletivo). Assim, para as instituições anfitriãs e os pesquisadores organizadores, trata-se de uma oportunidade para reafirmar uma posição de distinção na comunidade científica; para os cientistas e pesquisadores periféricos que frequentam os eventos como participantes regulares, representam uma oportunidade de ampliar sua própria legitimidade por meio da participação em uma arena cognitiva e política reconhecida.

Por sua vez, os eventos periféricos podem se caracterizar pela emulação ou inovação em relação aos eventos centrais. No primeiro caso, os eventos se baseiam em um modelo de organização tradicional e na discussão de temas já consagrados pelos eventos centrais. Procuram reproduzir em espaços periféricos algumas das condições e características que constituem a legitimidade e reputação dos eventos já estabelecidos. Quando bem-sucedidos, estes eventos periféricos demonstram a consolidação da reputação de instituições ou associações, ou ainda o amadurecimento de uma disciplina em um dado espaço geográfico. No segundo caso, os eventos se organizam em torno de pesquisadores, instituições ou temas marginais ou negligenciados nos espaços centrais da comunidade científica. Neste sentido, costumam ser menos estruturados do ponto de vista da organização e menos rígidos do ponto de vista hierárquico e reputacional. Sobretudo, em sentido inverso aos eventos emuladores, evidenciam as comunidades e os temas menos privilegiados nos espaços científicos centrais. 
FIGURA 3

EVENTOS CENTRAIS E PERIFÉRICOS

E O PAPEL DOS CIENTISTAS

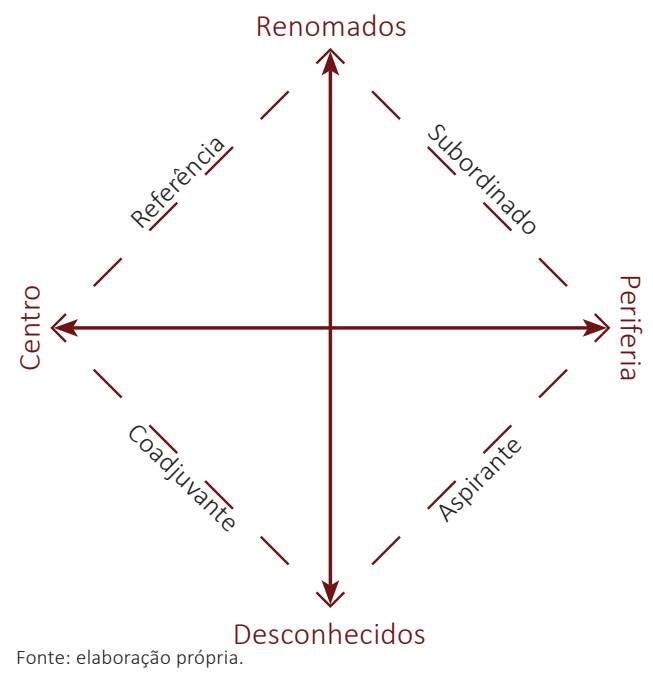

A rígida hierarquia dos eventos, sua excessiva formalização e a marginalização de temas e espaços periféricos demonstram algumas limitações dos eventos científicos. Em outras palavras, a dupla estratificação dos eventos científicos apresenta consequências indesejadas e nem sempre percebidas: internamente, estimulam o conservadorismo e as redes denominadas por David Travis e Harry Collins (1991) como redes de "old boys" (cientistas com as mesmas concepções em determinada área de pesquisa e um espírito de compadrio); do ponto de vista espacial, podem implicar em segregação ou mimetismo. Diante disso, diferentes inovações vêm sendo propostas e implementadas em relação ao formato dos eventos, sua localização, comunicação interna e externa, e processos de governança. Nesta direção é possível citar a ampliação da publicação eletrônica dos trabalhos (Kelly, 1998), a tendência de organização de conferências multidisciplinares (Zusman, 2011), o uso de mídias sociais para comunicação e divulgação (Wilkinson et alii, 2015) e a integração do público leigo ou comunidades de pacientes (Fixdal, 1997). Essas inovações buscam contornar a forte estratificação existente nos eventos científicos.

Do ponto de vista do Programa Mertoniano, os eventos científicos exprimem o funcionamento da comunidade científica. Os eventos científicos têm a função de promover cientificamente os cientistas e suas instituições. Caracterizam-se por um duplo processo de hierarquização: 


\section{i. Dimensão interna: Pirâmide Reputacional; e \\ ii. Dimensão externa: diferenciação espacial.}

Fica estabelecida uma homologia entre a posição que o cientista ocupa no evento e a localização de sua vinculação institucional. Servem, ao mesmo tempo, para reforçar tanto a reputação dos cientistas mais renomados como das instituições mais reconhecidas. Isso significa que os cientistas mais influentes vêm das regiões onde se realizam os eventos mais importantes em cada área. Neste sentido, os eventos científicos refletem a forma como a comunidade científica está estruturada. A autonomização da comunidade científica tem como consequência o lastreamento social e espacial. Pode-se dizer, portanto, que os eventos materializam socialmente e espacialmente o efeito Mateus.

\section{O programa discursivo: os círculos persuasivos}

A guinada discursiva da abordagem sociológica da ciência se caracteriza pela preocupação com a produção e circulação dos enunciados. Nesta perspectiva, o trabaIho científico passa a ser considerado como atividade literária (Callon, 1998). Afinal, os estudos de laboratório revelaram que a maior parte do tempo os cientistas estão lendo e escrevendo. Mais precisamente, que os cientistas não trabalham sobre a natureza, mas sobre inscrições produzidas no laboratório. Assim, a atenção sociológica se desloca para os processos de produção, interpretação, discussão e tradução dos enunciados observacionais em enunciados universais. É através da estabilização de equivalências discursivas entre rede de instrumentos, protocolos de experiência, competências cognitivas, inscrições etc., que se estabelece a adequação entre o discurso sobre o mundo e o mundo. Trata-se, portanto, de saber como os eventos científicos operam no processo por meio do qual um enunciado produzido localmente num laboratório é redistribuído universalmente pela sociedade.

A guinada discursiva está relacionada ao progressivo questionamento da perspectiva mertoniana de cunho normativo, que acabou gerando gradativamente novas vertentes analíticas complementares. Assim, os sociólogos da ciência deixaram de examinar exclusivamente a comunidade científica, passando a problematizar o conteúdo da ciência (Bloor, 1976; Shapin, 1979) e, mais recentemente, as práticas cotidianas dos cientistas e engenheiros (Latour \& Woolgar, 1997; Callon, 1986; Latour, 2000). Ao acompanhar essas mudanças de interesse de pesquisa, também se opera uma mudança nas explicações sociológicas: em linhas gerais, ocorre um deslocamento dos valores e das normas para a determinação social do conhecimento e, 
por fim, para os aspectos discursivos da atividade científica. Neste último sentido, destacam-se os "estudos de laboratório", isto é, a apreensão etnográfica da atividade científica e o conjunto de trabalhos reunidos em torno da Teoria Ator-Rede de autores como Bruno Latour, Michel Callon e John Law.

De acordo com a Teoria Ator-Rede os mundos material e social são o "efeito continuamente criado pela teia de relações nas quais eles estão localizados" (Law, 2009: 141). Dessa forma, esta abordagem se dedica ao estudo das relações materiais e discursivas de elementos heterogêneos - objetos, máquinas, sujeitos, organizações, ideias etc. -, que constituem os enunciados científicos, os artefatos tecnológicos e os arranjos sociais. Essas relações são analisadas simetricamente, ou seja, considerando atores humanos e não humanos, a partir dos mesmos critérios e importância epistemológica (Mattedi, 2004). O cientista se apresenta nesta perspectiva como "construtor de fatos" que, a partir das atividades do laboratório, agrupa uma rede de diferentes elementos - inscrições, dados, relatórios, artigos - que podem definir e redefinir a natureza e a sociedade. Assim, os cientistas não descobrem fatos científicos, mas os constroem a partir de suas atividades cotidianas. Portanto, a ciência resulta de disputas retóricas entre seus praticantes.

A noção de credibilidade é um elemento importante para compreender este fenômeno. Mais do que o reconhecimento individual, relacionado à estrutura reputacional, a credibilidade pode ser atribuída aos pesquisadores, mas também aos resultados de pesquisa. Refletem, portanto, a expectativa de produção futura de novos dados e teorias científicas. Para descrever este fenômeno, Bruno Latour e Steve Woolgar (1997) propõem a ideia de círculo de credibilidade: para os autores, a atividade científica é um processo constante e cíclico de conversão de um tipo de capital em outro. Assim, no contexto do laboratório, cientistas frequentemente transformam recursos financeiros e simbólicos em dados e artigos; por sua vez, dados e artigos se converterão em credibilidade que, finalmente, garante o acesso a novos recursos e assim sucessivamente. Os eventos se inserem neste círculo de credibilidade justamente por serem um espaço para o convencimento e a negociação dos fatos e dados científicos, ou seja, uma arena para a construção da credibilidade.

Neste sentido, os eventos constituem um ponto de passagem obrigatório na translocalização das competências performadas nos laboratórios. Ao operar como ator e como rede, um evento científico transforma a comunicação numa interação simultaneamente regular e móvel. Enquanto rede, os eventos científicos funcionam como um fórum que define quem são os cientistas e que tipo de associação ele pode efetuar; como ator, os eventos científicos operam como porta-vozes de interesses e recursos. Isso significa que os eventos científicos fixam relações, mas 
também permitem o deslocamento de enunciados. Dessa forma, os eventos possibilitam que a realidade seja reunida, trabalhada e transladada. Assim, ao mesmo tempo em que o evento produz as condições de comunicação do autor, a comunicação do autor da comunicação produz o evento. Ao articular simetricamente elementos heterogêneos os eventos científicos operam como:

i. Evento como rede: centro de tradução; e

ii. Evento como ator: intermediário.

FIGURA 4

OS EVENTOS DO PONTO DE VISTA

DA TEORIA ATOR-REDE

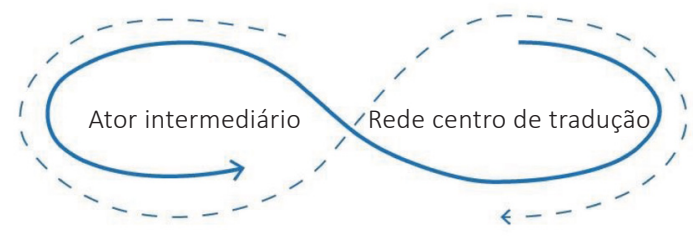

Fonte: elaboração própria.

Como centro de tradução, os eventos científicos constituem uma rede de transformações que agrega inscrições ao mundo por meio de relações híbridas. Mobilizam e articulam uma grande quantidade de entidades que participam ativamente da circulação dos fatos científicos. Reúnem elementos orais, escritos e pictóricos muito heterogêneos como, por exemplo, resumos, financiamentos, argumentos, dados, apresentações em PowerPoint, fotografias, mapas, tabelas, diagramas, filmes, registros acústicos, observações visuais, ilustrações, modelos 3D etc., que participam de maneira ativa, silenciosa e invisível da estabilização dos fatos científicos. Essa articulação discursiva recombina e sobrepõe instrumentos, indivíduos, instituições, entidades visando convencer o ouvinte da validade e visibilidade dos fatos científicos; permite que os enunciados sejam associados a uma entidade ou a outras inscrições. Desta forma, convertem uma simples declaração conjectural num argumento incontestável, que servirá de base para o trabalho de outros pesquisadores.

Enquanto intermediário, os eventos científicos possibilitam a circulação dos fatos científicos. Mais precisamente, constituem uma etapa crucial do processo de consolidação e disseminação do conhecimento produzido nos laboratórios (Rowley-Jolivet, 2011). Por um lado, os eventos científicos podem ser vistos como uma espécie de extensão das fronteiras do laboratório; por outro, ao tornar as pesquisas visíveis, quebram o hermetismo do trabalho de laboratório (Latour, 2006). Desta forma, os eventos científicos permitem que os fatos científicos sejam validados ou descartados diretamente através da circulação de argumentos, opiniões ou comentários. 
Servem para marcar as descobertas e para dar visibilidade à expertise desenvolvida por um grupo de pesquisa. Caracterizam-se e se diferenciam pelos tipos de associações e intermediações que o tornam possível. Portanto, os eventos científicos permitem que os fatos científicos saiam do laboratório, sejam negociados coletivamente, ganhem visibilidade, e se materializem no contexto social.

Ao traduzirem e intermediarem interesses, os eventos científicos efetuam um duplo círculo persuasivo. Por um lado, potencializam a capacidade associativa dos enunciados científicos aumentando sua visibilidade, por outro, diminuem as controvérsias reforçando a capacidade de negociação de seus porta-vozes. Essa dupla capacidade de convencimento discursivo dos eventos científicos converte a posição de cada participante no evento em ponto de chegada ou ponto de partida da comunicação. O destino do que cada participante enuncia depende dos interesses, projetos, valores, expectativas, o conhecimento de outros participantes que consegue mobilizar. Se o pesquisador desfruta de muita credibilidade, a comunicação é o ponto de chegada da estabilização de um fato científico; mas, se o pesquisador possui pouca credibilidade, o evento constitui o ponto de partida. Isso amplia o alcance dos enunciados e a credibilidade dos cientistas. Portanto, os eventos científicos fazem com que os enunciados se tornem um ponto de passagem obrigatório.

Para transladar uma substância do laboratório para um auditório, os eventos científicos efetuam uma mediação entre falantes e ouvintes. Essa mediação depende da atenção que a apresentação consegue despertar na audiência especializada. Aqui, o desafio consiste em chamar atenção do maior número de participantes, o que depende da capacidade de persuasão. A escolha do conteúdo, a estrutura da apresentação, a linguagem e os recursos semióticos são delimitados por diversas camadas discursivas: quem comunica, o que comunica, para quem comunica, por que meio, e com que propósitos (Ventola, Shalom \& Thompson, 2002). Porém, cada camada se baseia em estratégias interpessoais de audição e visualização que tendem a variar segundo a composição do público. Ao visarem o fortalecimento da capacidade de convencimento dos enunciados, os eventos medeiam e suportam três modalidades retóricas predominantes:
i. Enunciação oral;
ii. Enunciação audiovisual; e
iii. Enunciação escrita.

A Enunciação oral constitui a mediação mais difundida. Trata-se de um discurso didático visando persuadir os ouvintes da plausibilidade dos enunciados e, consequentemente, convencer a incorporá-los à prática (Carther-Thomas \& Rowley-Jo- 
vilet, 2003). Frequentemente, as exposições orais são estruturadas em função do conteúdo e dos ouvintes. Ao considerar o tempo de apresentação, a modalidade oral diferencia-se em dois gêneros:

i. Discurso lido (mais de 30 minutos): em conferências e mesas temáticas onde o tempo é maior, a apresentação costuma ser lida pelo modo impessoal;

ii. Discurso falado (até 15 minutos): em grupos de trabalho, onde o tempo é reduzido e a apresentação é preparada de modo pessoal.

Por um lado, depende muito da capacidade de oratória do pesquisador; por outro, está relacionada à língua utilizada. Neste sentido, atualmente, as apresentações mais influentes são aquelas efetuadas nos eventos científicos em inglês, porque permitem uma maior circulação dos enunciados. Portanto, quanto mais fortes as alianças do enunciado maior a atenção da apresentação oral.

A Enunciação audiovisual complementa a exposição oral como suporte técnico para sua apresentação. Trata-se das ilustrações, gráficos, tabelas, gravações de áudio, vídeos, animações ou outros recursos que são exibidos como forma de esclarecer e simplificar o que é falado pelos cientistas, fortalecendo o efeito retórico das apresentações. Portanto, do retroprojetor ao Prezi, passando pelo PowerPoint, os recursos tecnológicos de visualização das ideias se tornam sempre mais presentes e decisivos no processo de persuasão. Em outras palavras, são enunciados positivos na forma de recursos gráficos, que buscam aumentar a impessoalidade e diminuir as possíveis controvérsias em torno de uma afirmação. Assim, a crença simples na palavra do expositor é substituída pelo exame de figuras, números e legendas, tornando a discussão mais técnica: para discordar das premissas de uma exposição, um ouvinte precisa contestar os dados em sua forma simplificada (Latour, 2000). Por isso, são cada vez menos usuais apresentações sem recursos audiovisuais.

A Enunciação escrita é a modalidade retórica mais durável e mais propensa à circulação em diferentes espaços. São as publicações em anais (proceedings) de eventos, podendo ser constituídas por resumos, resumos expandidos ou artigos completos. Visam documentar a contribuição dos cientistas para o evento, em formato impresso, eletrônico ou ambos. Neste aspecto, são uma representação ampla das atividades que constituem um evento, ou seja, sua face mais visível para o público externo. Quando comparadas com artigos publicados em revistas acadêmicas, essas modalidades escritas costumam contar com uma menor capacidade de persuasão, porque, frequentemente, apresentam resultados de pesquisa parciais ou inconclusivos, e por estarem submetidas a um processo de avaliação por pares menos estruturado. 
FIGURA 5

AS MODALIDADES DE PERSUASÃO

EM EVENTOS CIENTÍFICOS

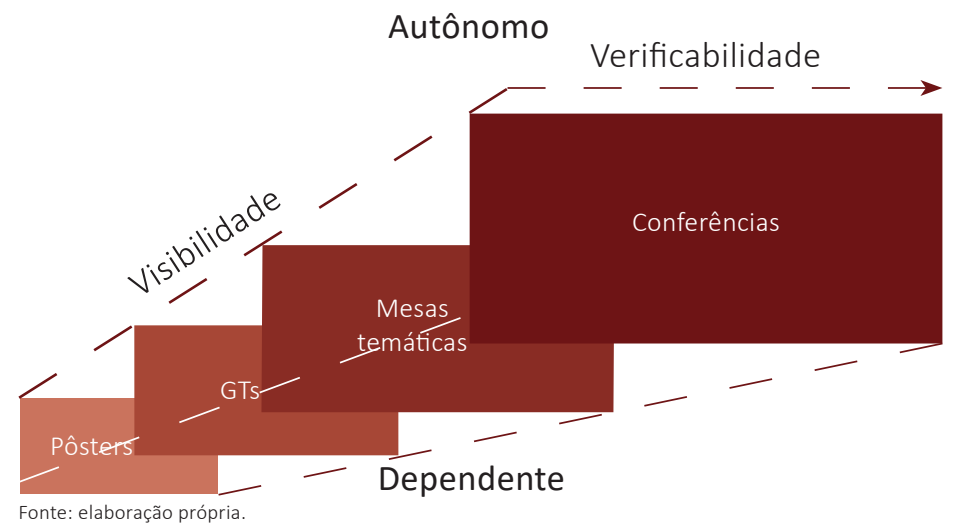

Assim, se aproximam em termos funcionais da grey literature. Todavia, trata-se de prática distintiva dos eventos científicos consolidados e estabelecidos.

Analisadas em conjunto, essas três modalidades retóricas exprimem o funcionamento dos eventos científicos como espaços de persuasão e negociação. De forma particular, evidenciam as diferentes estratégias empregadas pelos cientistas para efetivar o duplo círculo persuasivo. Do ponto de vista da organização social da produção científica, representam a extensão da rede originada nos laboratórios e universidades, bem como a gradativa incorporação de novos elementos técnicos e retóricos. Do ponto de vista cognitivo, se caracterizam como espaços exemplares, que determinam os modelos teóricos e métodos aceitos, e as formas legítimas de persuasão em uma comunidade científica. Por fim, em relação à distribuição espacial dos enunciados científicos, possibilitam que os enunciados científicos se tornem "móveis imutáveis" (Latour, 1995), ou seja, elementos simplificados que extrapolam seu contexto de produção originário, atuando em esferas sociais mais amplas. Neste sentido, são fundamentais para o estabelecimento da credibilidade científica.

\section{Considerações finais}

Embora os eventos científicos desempenhem um papel central na comunicação científica, são muito pouco conhecidos na própria comunidade científica. De forma geral, pode-se dizer que os eventos científicos divulgam as descobertas científicas e promovem os cientistas. Neste sentido, os eventos científicos formam um contexto 
ao mesmo tempo social, cognitivo e territorial, que varia segundo a magnitude e a intensidade do evento. Trata-se, portanto, de explicar quais são as condições sociais que tornam possíveis os eventos científicos.

Do ponto de vista do Programa normativo, os eventos científicos compreendem uma etapa no processo de integração da comunidade científica. O foco analítico desta abordagem se concentra na posição social dos participantes e na localização geográfica dos eventos. Isso significa que os eventos científicos são produzidos e reproduzem uma estratificação ao mesmo tempo social (entre cientistas) e espacial (entre comunidades). O efeito combinado deste processo estabelece uma hierarquização chamada duplo efeito Mateus:

i. reflete o reconhecimento: diferencia os cientistas em função de sua posição na estrutura de comunicação; e

ii. diferencia espacialmente: estrutura os eventos em função da localização da sua realização.

Verifica-se que quanto maior a abrangência espacial do evento, em termos de edições e de participantes, maior a institucionalização da comunidade científica e, consequentemente, mais hierarquizada as relações entre os cientistas. Consequentemente, o aumento da diferenciação provoca o aumento da competição.

Já do ponto de vista do Programa discursivo, os eventos científicos constituem uma das mediações por meio da qual os fenômenos locais se tornam universais. Por isso a ênfase analítica recai sobre os conteúdos das apresentações. Os eventos científicos, ao operarem, simultaneamente, como atores e como rede instauram um duplo círculo persuasivo:

i. os eventos científicos são "controvérsias institucionalizadas": espaços onde a resistência dos enunciados é testada; e

ii. uma "assembleia de negociação": ambientes onde são formadas alianças entre intermediários.

A resistência dos enunciados depende da posição do evento na rede técnico-científica e da posição dos participantes no evento. Após sua apresentado num evento científico, um enunciado pode se tornar uma caixa-preta, ou, ao contrário, suas características serem questionadas, rearranjadas e modificadas. Portanto, os eventos científicos afetam a redistribuição dos enunciados científicos na medida em que mudam o ritmo como eles são transladados dos laboratórios para o contexto social. 
Os subsídios extraídos da aplicação destas duas abordagens indicam um paradoxo intrigante: quanto maior o evento, menor a inovação. É que o aumento do número de participantes não acompanha, proporcionalmente, a apresentação de novas descobertas. Por um lado, a atenção acaba se dispersando devido à fragmentação das questões tematizadas provocada pela diminuição da seletividade; por outro, a comunicação acaba sendo afetada pela adoção de um jargão sempre mais obscuro, típico do "compadrio" acadêmico. É que, frequentemente, em função das disputas por visibilidade entre grupos de interesses, chicanas teóricas e esoterismo temático os grupos se fecham neles mesmos. O custo cognitivo deste processo pode ser o obscurecimento de algumas descobertas e, portanto, a deformação do processo de comunicação. O efeito combinado desses fatores é que os eventos científicos se tornam cada vez mais frequentes, porém cada vez menos importantes. Isso significa que, paradoxalmente, quanto mais superpovoado um evento, mais difícil se torna a comunicação.

O padrão de organização dos eventos científicos não somente distorce a comunicação, mas também bloqueia a renovação devido a formação de uma eventolarquia. Essa eventolarquia é constituída por uma casta acadêmica que controla a burocracia organizadora e o comitê científico dos eventos científicos, assemelhada a uma oligarquia. Neste sentido, as questões debatidas nos eventos científicos, seu direcionamento cognitivo e posicionamento político costumam refletir os interesses dos pesquisadores que controlam burocraticamente os eventos científicos. Assim, os eventos parecem ser um caso particular da lei de ferro das oligarquias, originalmente proposta por Robert Michels, quando a reputação dos pesquisadores e sua capacidade de persuasão denotam um papel importante na determinação da elite dirigente. Por fim, o padrão de organização dos eventos permite supor que os privilégios, o prestígio e a autoridade tendem a se consolidar em torno de indivíduos e grupos que pretendem conservá-los, ao mesmo tempo em que são disputados por atores emergentes, com maior ou menor sucesso.

A abordagem sociológica dos eventos científicos evidencia um componente deliberadamente reflexivo. Afinal, ao analisar-se os eventos científicos, o tema é frequentemente considerado a partir das abordagens mais recorrentes nos próprios eventos científicos que tratam da ciência. Porém, esta é uma reflexividade restrita, na medida em que não se autoproblematiza no âmbito dos próprios eventos. Em outras palavras, os eventos científicos representam, curiosamente, um tema relativamente negligenciado e desconhecido pelos próprios cientistas. Em particular, representa uma lacuna significativa no campo da sociologia da ciência e dos estudos sociais da ciência e da tecnologia. Desse modo, analisar os mecanismos sociológicos que caracterizam os eventos científicos é uma forma de aumentar a compreensão 
não apenas sobre os processos de comunicação científica, mas ainda de exploração do fenômeno a partir de uma postura reflexiva, que não trata as ciências sociais como um caso especial da prática científica.

Todavia, o estudo dos eventos científicos encontra-se ainda num estágio inicial. Um dos desafios evidencia a dificuldade de aquisição empírica das enunciações orais e audiovisuais, ou seja, a descrição das práticas rotineiras dos eventos. Assim, é preciso considerar os procedimentos metodológicos necessários e a possível posição ambígua de observador participante. Interessa também saber se os eventos científicos podem ser analisados como os textos científicos. Neste sentido, cabe avaliar a aplicabilidade e a validade das ferramentas metodológicas dos campos da cientometria e da bibliometria, bem como o uso de metodologias de análise de discurso. Por fim, é possível considerar a elaboração de ferramentas para a avaliação dos eventos, desde o ponto de vista do financiamento e da viabilidade financeira até a questão de sua eficácia para o avanço da ciência, cogitando os dilemas de hierarquização e constituição de oligarquias acadêmicas. Afinal, os eventos representam diferentes dimensões sociais da comunicação científica, muitas delas ainda inexploradas.

\section{Referências}

ALEIXANDRE-BENAVENT, Rafael; GONZÁLEZ-ALCAIDE, Gregorio; MIGUEL-DASIT, Alberto; NAVARRO-MOLINA, Carolina; VALDERRAMA-ZURIÁN, Juan C. Full-text publications in peer-reviewed journals derived from presentations at three ISSI conferences. Scientometrics, v. 80, p. 407, 2009.

BEN-DAVID, Joseph. O papel do cientista na sociedade: um estudo comparativo. São Paulo: Editora Universidade de São Paulo, 1974.

BLOOR, David. Knowledge and social imagery. Chicago (IL): University of Chicago Press, 1976.

BOURDIEU, Pierre. Homo academicus. Florianópolis: Editora UFSC, 2011.

. Les usages sociaux de la science: pour une sociologie clinique du champ cientifique. Paris: Inra Editions, 2009.

Science de la science et reflexivité. Paris: Raisons d'Agir Éditions, 2001.

. Le champ scientific. Actes de La recherché en Sciences Sociales, v. 2, n. 3, p. 88-104, 1976. 
BRITISH MEDICAL JOURNAL. The First International Malaria Congress, v. 2, n. 3386, p. 970-971, 1925.

Some elements of a sociology of translation: domestication of the scallops and the fishermen of St. Brieuc Bay. In: LAW, J. (Org.). Power, action and belief: a new sociology of knowledge?, p. 196-223. London: Routledge, 1986.

—. Défense et illustration des recherches sur la science. In: JURDANT, B. (Org.). Impostures scientifiques, p. 253-267. Paris: La Découverte, 1998.

CALLON, Michel. Some Elements of a Sociology of Translation: Domestication of the Scallops and the Fishermen of St Brieuc Bay. in LAW, John (ed.) Power, Action and Belief: A New Sociology of Knowledge, Londres: Routledge \& Kegan Paul, 1986.

CARPI, Anthony; EGGER, Anne. The process of science. New Canaan (CT): Visionlearning, 2009.

CARTHER-THOMAS, Shirley; ROWLEY-JOVILET, Elizabeth. Analysing the scientific conference presentation (CP), a methodological overview of multimodal genre. Asp La Revue du Groupe d'Études et de Recherches en Anglais de Spécialité (Geras), v. 39, p. 59-72, 2003.

COLE, Stephen. Merton's contribution to the sociology of science. Social Studies of Science, v. 34, n. 6, p. 829-844, 2004.

COLLINS, Harry M.; PINCH, Trevor J. O Golem: o que você deveria saber sobre ciência. São Paulo: Editora Unesp, 2003.

DROTT, M. Carl. Reexamining the role of conference papers in scholarly communication. Journal of the American Society for Information Science, v. 46, n. 4, p. 299305, 1995.

EVERTS, Sarah. When science went international. Chemical \& Engineering News, 2010. Disponível em: <https://pubs.acs.org/cen/science/88/8836sci1.html>. Consultado em: 08 Maio 2017.

FIXDAL, Jon. Consensus conferences as "extended peer groups". Science and Public Policy, v. 24, n. 6, p. 366-376, 1997.

GARFIELD, Eugene. What is the primordial reference for the phrase "publish or perish"? The Scientist, v. 10, n. 12, p. 11, 1996.

GLÄNZEL, Wolfgang; DEBACKERE, Koenraad; THIJS, Bart; SCHUBERT, András. A concise review on the role of author self-citations in information science, bibliometrics and science policy. Scientometrics, v. 67, n. 2, p. 263-277, 2006. 
GRIBBIN, John. The fellowship: Gilbert, Bacon, Wren, Newton, and the story of a scientific revolution. London: Penguin Books, 2007.

HAGSTROM, Warren. The scientific community. New York: Basic Books, Inc., 1965.

HAYASHI, Maria C. P. I.; GUIMARÃES, Vera A. L. A comunicação da ciência em eventos científicos na visão de pesquisadores. Em Questão, v. 22, n. 3, p. 161-183, 2016.

HESSELS, Laurens K.; van LENTE, Harro. Re-thinking new knowledge production: a literature review and a research agenda. Research Policy, v. 37, n. 4, p. 740-760, 2008.

HERMENEGILDO, Manuel V. Conferences vs. journals in CS, what to do? Evolutionary ways forward and the ICLP/TPLP Model. Position paper for Dagstuhl meeting 12452. Dagstuhl (DE): Publication Culture in Computing Research, 2012.

IHDE, Aaron. The Karlsruhe Congress: a centennial retrospect. Journal of Chemical Education, v. 38, n. 2, p. 83-86, 1961.

KELLY, Julia A. Scientific meeting abstracts: significance, access, and trends. Bulletin of the Medical Library Association, v. 86, n. 1, p. 68-76, 1998.

KNORR-CETINA, Karen. The manufacture of knowledge: an essay on the constructivist and contextual nature of science. Oxford; New York: Pergamon Press, 1981.

LATOUR, Bruno. Changer de societé, refaire de la sociologie. Paris: La Découverte, 2006.

. Ciência em ação: como seguir cientistas e engenheiros sociedade afora. São Paulo: Editora Unesp, 2000.

. The "pedofil" of Boa Vista: a photo-philosophical montage. Common Knowledge, v. 4, p.144-187, 1995.

LATOUR, Bruno; WOOLGAR, Steve. A vida de laboratório: a produção dos fatos científicos. Rio de Janeiro: Relume Dumará, 1997.

LAW, John. Actor network theory and material semiotics. In: TURNER, B. (Ed.). The New Blackwell companion to social theory. Malden (MA): Blackwell Publishing, 2009.

LISÉE, Cynthia; LARIVIÈRE, Vincent; ARCHAMBAULT, Éric. Conference proceedings as a source of scientific information: a bibliometric analysis. Journal of the American Society for Information Science and Technology, v. 59, n. 11, p. 1776-1784, 2008. 
LYNCH, Michael. Art and artifact in laboratory science: a study of shop work and shop talk in a research laboratory. London; Boston (MA): Routledge; Kegan Paul, 1985.

MARTINS, Waister S.; GONÇALES, Marcos A.; LAENDER, Alberto H .F.; ZIVIANI, Nivio. Assessing the quality of scientific conferences based on bibliographic citations, Scientometrics, v. 83, n. 1, p. 133-155, 2010.

MATTEDI, Marcos A. Dilemas da simetria entre contexto social e conhecimento: a redefinição das modalidades de abordagem sociológica do problema do conhecimento. Política \& Sociedade, v. 3, n. 4, p. 41-79, 2004.

MATTEDI, Marcos A.; SPIESS, Maiko R. A avaliação da produtividade científica. História, Ciência, Saúde-Manguinhos, v. 24, n. 3, p. 623-643, 2017.

— Modalidades de regulação da comunidade científica: uma comparação entre as interpretações normativa, cognitiva e transacional dos processos de integração da comunidade científica. Educação \& Sociedade, v. 31, n. 110, p. 73-111, 2010.

MEADOWS, A. J. A comunicação científica. Brasília: Briquet Lemos Livros, 1999.

MERTON, Robert K. A ambivalência sociológica e outros ensaios. Rio de Janeiro: Zahar, 1979.

- Sociologia: teoria e estrutura. São Paulo: Mestre Jou, 1970.

_. The Matthew effect in science. Science, v. 159, n. 3810, p. 56-63, 1968.

- Priority in scientific discovery. American Sociological Review, v. 22, n. 6, p. 635-659, 1957.

MICHELS, Carolin; FU, Jun-Ying. Systematic analysis of coverage and usage of conference proceedings in web of science. Scientometrics, v. 100, n. 2, p. 307-327, 2014.

MOMM, Christiane F. Conhecimento cientifico e território: as dimensões espaciais do Enanpur (Anpur) no Brasil, 1986-2013. Tese (Doutorado em Desenvolvimento Regional) - Programa de Pós-Graduação em Desenvolvimento Regional, Universidade Regional de Blumenau, 2017.

NYE, Mary. Introduction. In: NYE, M. J. (Org.). The question of the atom: from the Karlsruhe Congress to the First Solvay Conference, 1860-1911. Los Angeles (CA): Tomash Publishers, 1984.

POWELL, Walter W. Getting into Print: the decision making process in scholarly publishing. Chicago (IL): University of Chicago Press, 1985. 
PRICE, Derek John de Solla. Little science, big science. New York: Columbia University Press, 1963.

ROWLEY-JOLIVET, Elizabeth. ROWLY-JOLIVET, Elizabeth. The pivotal role of conference papers in the network of scientific communication. ASp [Online], v. 23, p. 179-196, 2011.

- The pivotal role of conference papers in the network of scientific communication. Asp La Revue du Groupe d'Études et de Recherches en Anglais de Spécialité (Geras), v. 23, p. 179-196, 1999.

SHAPIN, Steven. The politics of observation: cerebral anatomy and social interests in the Edinburgh phrenology disputes. The Sociological Review, V. 27, S1, p. 139$178,1979$.

SHAPIN, Steven; SCHAFFER, Simon. Leviathan and the air-pump: Hobbes, Boyle, and the experimental life. Princeton (NJ): Princeton University Press, 1985.

SILVEIRA, Murilo Artur Araújo da; BUFREM, Leilah Santiago; CAREGNATO, Sônia Elisa. Os eventos científicos, as relações de poder e as práticas dos pesquisadores. Transinformação, v. 27, n. 3, p. 199-208, 2015.

SÖDERQVIST, Thomas; SILVERSTEIN, Arthur M. Studying leadership and subdisciplinary structure of scientific disciplines. Scientometrics, v. 30, n. 1, p. 243-258, 1994.

STAFLEU, Frans. A century of botanical congresses. In: STARR, R. C. (Ed.). XI International Botanical Congress. Seattle (WA): University of Washington, Seattle, August 24-September 2, 1969. Proceedings, Washington (DC), XI International Botanical Congress, 1970.

TRAVIS, David; COLLINS, Harry M. New light on old boys: cognitive and institutional particularism in the peer review system. Science, Technology and Human Values, $v$. 16, n. 3, p. 322-341, 1991.

VARDI, Moshe Y. Conferences vs. journals in computing research. Communications of the ACM, v. 52, n. 5, p. 5, 2009.

VENTOLA, Eija; SHALOM, Celia; THOMPSON, Susan. The language of conferencing. New York: Peter Lang, 2002.

WEBSTER, Charles. New light on the invisible college the social relations of English science in the mid-seventeenth Century. Transactions of the Royal Historical Society, v. 24, p. 19-42, 1974. 
WILKINSON, Sarah E.; BASTO, Marnique Y.; PEROVIC, Greta; LAWRENTSCHUK, Nathan; MURPHY, Declan G. The social media revolution is changing the conference experience: analytics and trends from eight international meetings. BJU International, v. 115, n. 5, p. 839-846, 2015.

ZIMAN, John. Real science: what it is and what it means. Cambridge (UK): Cambridge University Press, 2000.

Postacademic science: constructing knowledge with networks and norms. Science Studies, v. 9, n. 1, p. 67-80, 1996.

ZUSMAN, Edie E. Restructuring of multidisciplinary scientific meetings improves valuable exchange of information. Neurosurgery, v. 69, n. 4, p. N16-N18, 2011. 
